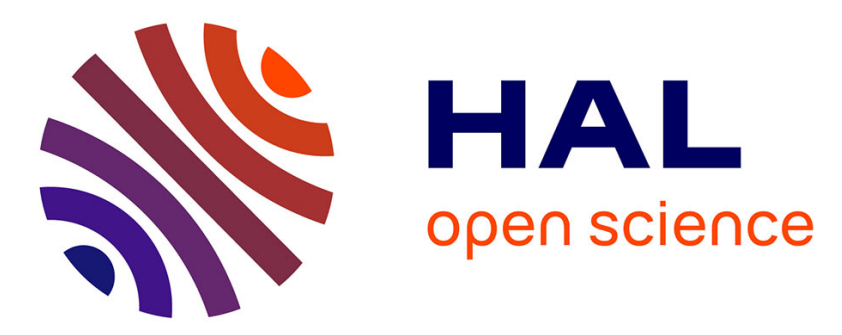

\title{
Optimized High Order Explicit Runge-Kutta-Nyström Schemes
}

Marc Duruflé, Mamadou N'Diaye

\section{To cite this version:}

Marc Duruflé, Mamadou N'Diaye. Optimized High Order Explicit Runge-Kutta-Nyström Schemes. ICOSAHOM 2016 - International Conference on Spectral and High-Order Methods, Jun 2016, Rio de Janeiro, Brazil. pp.599-612. hal-01403338v2

\section{HAL Id: hal-01403338 \\ https://hal.science/hal-01403338v2}

Submitted on 25 Oct 2017

HAL is a multi-disciplinary open access archive for the deposit and dissemination of scientific research documents, whether they are published or not. The documents may come from teaching and research institutions in France or abroad, or from public or private research centers.
L'archive ouverte pluridisciplinaire HAL, est destinée au dépôt et à la diffusion de documents scientifiques de niveau recherche, publiés ou non, émanant des établissements d'enseignement et de recherche français ou étrangers, des laboratoires publics ou privés. 


\title{
Chapter 1 \\ Optimized High Order Explicit Runge-Kutta-Nyström Schemes
}

\author{
Marc Duruflé and Mamadou N'diaye
}

\begin{abstract}
Runge-Kutta-Nyström (RKN) schemes have been developed to solve a non-linear ordinary differential equation (ODE) of the type $y^{\prime \prime}=f(t, y)$. In [4], the stability condition (the Courant-Friedrichs-Lewy or CFL) associated with these schemes have been studied for order 3, 4 and 5. In this paper, we extend this study for higher orders and we propose a new algorithm to compute numerically the CFL. By using this algorithm, we compute optimal coefficients for RKN schemes of orders $6,7,8$ and 10 which maximize the CFL. Herein, the obtained schemes are used to solve non-linear Maxwell's equations in 1-D.
\end{abstract}

\subsection{Introduction}

We consider the following ordinary differential equation (ODE)

$$
\left\{\begin{array}{l}
y^{\prime \prime}(t)=f(t, y(t)), \quad \forall t>0 \\
y(0)=y_{0} \\
y^{\prime}(0)=y_{0}^{\prime}
\end{array}\right.
$$

The unknown $y$ is vectorial, its size is equal to the number of the degrees of freedom of the system. The functional $f$ is known and describes the dynamics of the system. This kind of ODE appears naturally in mechanical systems when the damping terms are neglected, and also in non-linear wave equation. In order to solve this ODE,

Marc Duruflé

Magique-3D, Inria Centre de Recherche Bordeaux Sud-Ouest, 200 avenue de la vieille Tour, 33 405 Talence, FRANCE, e-mail: marc.durufle@inria.fr

Mamadou N'diaye

Laboratoire de Mathématiques et leurs Applications, University of Pau and Pays de L'Adour, avenue de l'université, 64012 Pau, e-mail: mamadou.ndiaye@univ-pau.fr

Magique-3D, Inria Centre de Recherche Bordeaux Sud-Ouest, 200 avenue de la vieille Tour, 33 405 Talence, FRANCE, e-mail: mamadou.ndiaye@inria.fr 
high-order Runge-Kutta-Nyström (RKN) schemes have been proposed (see [1]). They are attractive because they are explicit, one-step methods and can be applied to a non-linear operator $f$. A RKN scheme computes a discrete sequence $y_{n}$ and $y_{n}^{\prime}$, which are an approximations of $y$ and $y^{\prime}$ at time $t_{n}=n \Delta t$. The time step $\Delta t$ is assumed to be constant in this paper. A step of the RKN scheme is performed as follows:

$$
\left\{\begin{array}{l}
k_{i}=f\left(t_{n}+c_{i} \Delta t, \quad y_{n}+c_{i} \Delta t y_{n}^{\prime}+\Delta t^{2} \sum_{j} \bar{a}_{i, j} k_{j}\right), \\
y_{n+1}=y_{n}+\Delta t y_{n}^{\prime}+\Delta t^{2} \sum_{j} \bar{b}_{j} k_{j}, \\
y_{n+1}^{\prime}=y_{n}^{\prime}+\Delta t \sum_{j} b_{j} k_{j},
\end{array}\right.
$$

$k_{i}$ are intermediate vectors used to compute $y_{n+1}$ and $y_{n+1}^{\prime}$. The coefficients $\bar{a}_{i, j}, c_{i}, b_{i}, \bar{b}_{i}$ must satisfy the so-called order conditions such that the scheme is of order $r$ (see [1] for a detailed description of order conditions). When it is not mentioned, the subscripts $i$ and $j$ vary between 0 and $s-1$ where $s$ is the number of stages of the scheme. In this paper, only explicit schemes will be studied, the matrix $\bar{A}$ (associated with coefficients $\bar{a}_{i, j}$ ) is lower triangular, that is to say:

$$
\bar{a}_{i, j}=0, \text { if } j \geq i .
$$

The coefficients $\bar{a}_{i, j}, c_{i}, b_{i}, \bar{b}_{i}$ can be obtained from the coefficients of a classical Runge-Kutta scheme of order $r$. But this procedure leads to RKN schemes that are less efficient (see [1]). In this paper, we are concerned to find optimal coefficients $\bar{a}_{i, j}, c_{i}, b_{i}, \bar{b}_{i}$ that maximize the CFL number subject to the order conditions. Such an optimization has been done for RKN schemes of order 3, 4 and 5 in [4] and [5]. In this work, we achieved to find the best coefficients for order 6, 7, 8 and 10. We propose a new algorithm to compute numerically the CFL number (stability condition) with respect to the coefficients $\bar{a}_{i, j}, c_{i}, b_{i}, \bar{b}_{i}$.

The remainder of this paper is organized as follows: First, we recall the stability condition as initially proposed in [4]. Next, we describe the numerical algorithm we used to compute the CFL number. Then, we propose the optimal coefficients obtained for the different schemes. Finally, we present some numerical results to show the practical interest of these schemes.

\subsection{Stability condition}

The stability analysis is conducted for a linear functional $f$, which is then replaced by a matrix $A$ :

$$
f(t, y)=A y .
$$

By replacing $A$ by its symbol $\hat{A}$ (which will be equal to an eigenvalue of $A$ ), a step of RKN scheme can be written as:

$$
\left[\begin{array}{l}
y_{n+1} \\
w_{n+1}
\end{array}\right]=D\left(\Delta t^{2} \hat{A}\right)\left[\begin{array}{l}
y_{n} \\
w_{n}
\end{array}\right]
$$


where $D\left(\Delta t^{2} \hat{A}\right)$ is a $2 \times 2$ matrix depending on coefficients $\bar{a}_{i, j}, b_{i}, c_{i}, \bar{b}_{i}$. Let us note:

$$
z=\Delta t^{2} \hat{A}
$$

The vector $w_{n}$ is equal to:

$$
w_{n}=\frac{y_{n}^{\prime}}{\Delta t \hat{A}} .
$$

The RKN scheme is equal to:

$$
\left\{\begin{array}{l}
\Delta t^{2} k_{i}=z y_{n}+c_{i} z^{2} w_{n}+z \sum_{j} \bar{a}_{i, j} \Delta t^{2} k_{j} \\
y_{n+1}=y_{n}+z w_{n}+\sum_{i} \bar{b}_{i} \Delta t^{2} k_{i} \\
w_{n+1}=w_{n}+\frac{1}{z} \sum_{i} b_{i} \Delta t^{2} k_{i} .
\end{array}\right.
$$

From these relations, it can be remarked that the entries of the $2 \times 2$ matrix $D(z)$ are polynomials in $z$. The amplification factor $G(z)$ is defined as:

$$
G(z)=\text { Spectral radius of } D(z) .
$$

The stability condition is computed numerically by searching the first $z$ such that

$$
G(z)>1 \text {. }
$$

The square root of this first $z$ is defined as the CFL number:

$$
\text { CFL number }=\min _{z \leq 0}\{\sqrt{-z} \text { such that } G(z)>1\} .
$$

\subsection{Numerical method to compute the CFL}

The eigenvalues of the $2 \times 2$ matrix $D(z)$ are directly computed as:

$$
\lambda(D(z))=\frac{\operatorname{trace}(D(z)) \pm \sqrt{\operatorname{trace}(D(z))^{2}-4 \operatorname{det}(D(z))}}{2} .
$$

The amplification factor $G(z)$ is the maximal modulus of these two eigenvalues. From the computation of this amplification factor, the method used to compute the CFL is detailed in Algorithm 1. The computation of local maxima $z_{m}$ and of the final z such that $G(z)=1+\varepsilon$ is performed by using a bisection method. The first float $z_{0}$ is chosen small (we have chosen $z_{0}=-10^{-5}$ ), this first verification is needed because it happens that the amplification factor is decreasing at the origin, ie:

$$
G^{\prime}(0)<0
$$




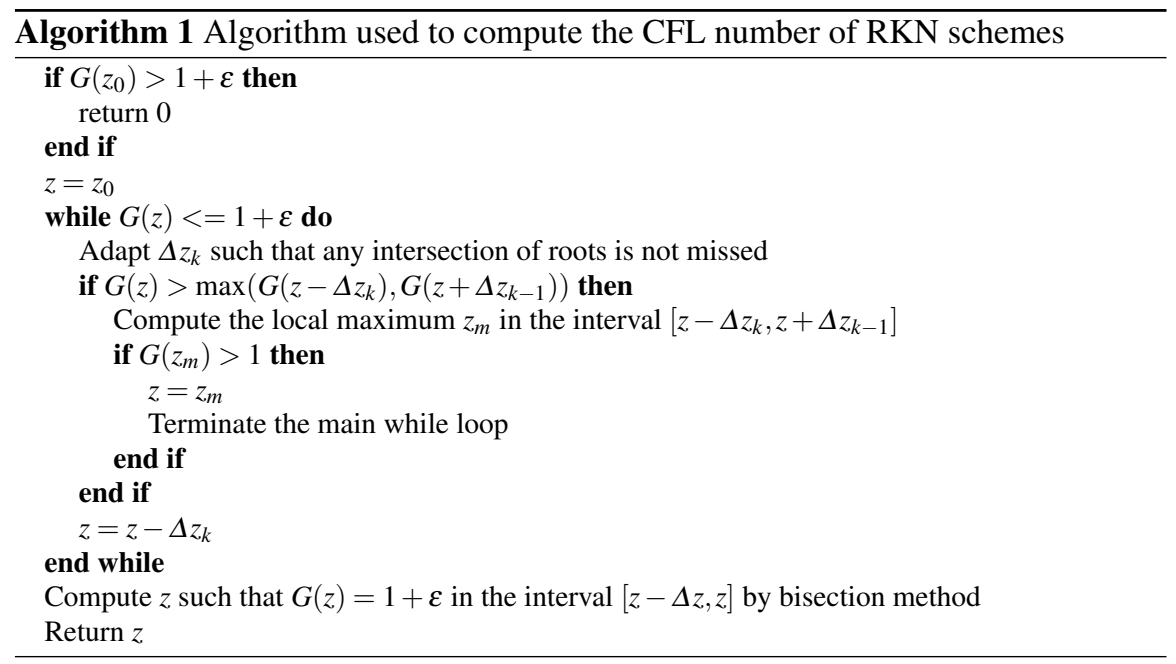

Hence for very small negative values of $z$, this amplification factor will be greater than one, leading to an unstable scheme. In this case, the time scheme will be

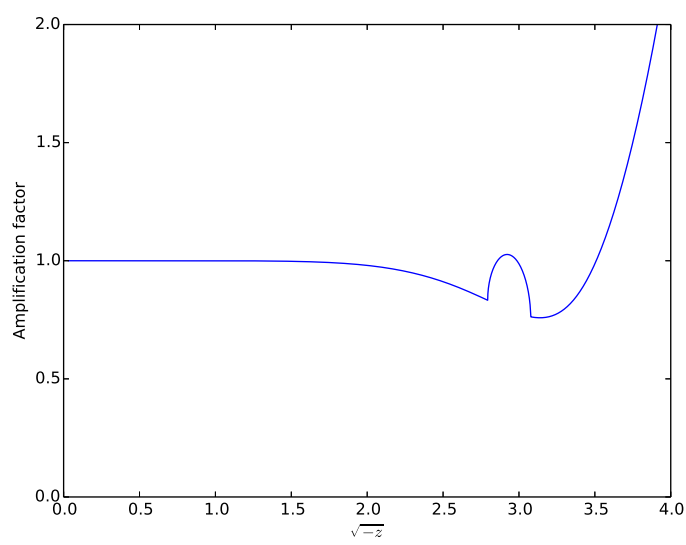

Fig. 1.1 Amplification factor $G(z)$ versus $\sqrt{-z}$ for a 6-th order RKN scheme, with the two free parameters equal to 0.0816464646464646 and 0.968757575757576 .

unconditionally unstable.

The step $\Delta z_{k}$ is chosen in an interval (we have selected $10^{-5} \leq \Delta z_{k} \leq 1$ ) such that the intersection of the two complex conjugate eigenvalues is not missed. This intersection occurs when the two eigenvalues get close to the real axis or when they already lie in the real axis. In Figure 1.1, the amplification factor is displayed for a 6th order RKN method. In this case, the CFL is equal to 2.858 because of 


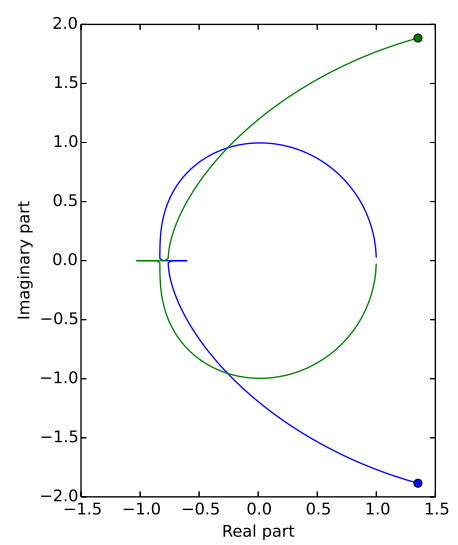

Fig. 1.2 Trajectory of the two eigenvalues of $D(z)$ for $\sqrt{-z} \in[0,4]$ for a 6-th order RKN scheme, with the two free parameters equal to 0.0816464646464646 and 0.968757575757576 .

the presence of a local maxima above 1 . It has been observed that usually the first local maximum (if present) occurs around $\sqrt{-z} \approx \pi$, the second maximum would occur around $2 \pi$, etc. In Figure 1.2, we have displayed the trajectory of the two eigenvalues of $D(z)$ for $\sqrt{-z} \in[0,4]$. These two eigenvalues start from the point $(1,0)$ they describe an approximate circle to reach a point close to $(-1,0)$. Then they move away from each other in the real axis, one reaches the local maximum, and the two eigenvalues get back until reaching another intersection in the real axis. Finally, they are describing a kind of hyperbole in the complex plane. With a variable $\Delta z_{k}$, we are able to compute the CFL with a reasonable number of evaluations of the amplification factor. Finally, $\varepsilon$ is taken equal to $2 \cdot 10^{-13}$ for a double precision computation.

\subsection{Optimization with a minimal number of stages}

In this section, coefficients of RKN schemes are optimized to maximize the CFL number. We consider here only schemes with a minimal number of stages (s).

\subsubsection{Order $2(s=1)$}

For example, to obtain a second-order scheme, it is sufficient to satisfy

$$
\sum_{i} b_{i}=1, \quad \sum_{i} b_{i} c_{i}=\frac{1}{2}, \quad \sum_{i} \bar{b}_{i}=\frac{1}{2} .
$$


Therefore, a one-stage scheme can be obtained:

$$
\bar{A}=(0), \quad c=\left(\frac{1}{2}\right), \quad b=(1), \quad \bar{b}=\left(\frac{1}{2}\right),
$$

this scheme can be written as:

$$
\left\{\begin{array}{l}
k_{0}=f\left(t^{n}+\frac{\Delta t}{2}, y_{n}+\frac{\Delta t}{2} y_{n}^{\prime}\right), \\
y_{n+1}=y_{n}+\Delta t y_{n}^{\prime}+\frac{\Delta t^{2}}{2} k_{0}, \\
y_{n+1}^{\prime}=y_{n}^{\prime}+\Delta t k_{0} .
\end{array}\right.
$$

This scheme require only one evaluation of $f$ (i.e. a matrix-vector product if $f$ is linear) at each time step, which is equivalent to the cost of the classical second-order scheme (recalled below). When $f$ is linear (replaced by a matrix $A$ ), the stability condition of this RKN scheme is:

$$
\Delta t \leq \frac{2}{\sqrt{\|A\|_{2}}} .
$$

This is exactly the same CFL as the classical second-order scheme:

$$
\frac{y_{n+1}-2 y_{n}-y_{n-1}}{\Delta t^{2}}=f\left(t_{n}, y_{n}\right) .
$$

Therefore, the second-order Runge-Kutta-Nyström (RKN) scheme is optimal.

\subsubsection{Orders 3, 4 and 5}

For orders 3, 4, 5, we have found the same optimal coefficients for RKN schemes as in [4]. These coefficients are recalled below.

Order $3(s=2)$ : A third-order RKN scheme with 2 stages is given as:

$$
\begin{array}{ll}
c_{0}=\alpha, \quad c_{1}=\frac{2-3 \alpha}{3-6 \alpha}, & b_{0}=\frac{\frac{c_{1}}{2}-\frac{1}{3}}{c_{0}\left(c_{1}-c_{0}\right)}, \quad b_{1}=1-b_{0}, \\
\bar{b}_{0}=\frac{\frac{c_{1}}{2}-\frac{1}{6}}{c_{1}-c_{0}}, \quad \bar{b}_{1}=\frac{1}{2}-\bar{b}_{0}, & \bar{a}_{1,0}=\frac{1}{6 b_{1}},
\end{array}
$$

$\alpha$ is a free parameter, a maximal CFL of 2.498 is obtained for $\alpha=\frac{3-\sqrt{3}}{6}$. 
Order $4(s=3)$ : A fourth-order RKN scheme with 3 stages is given as:

$$
\begin{aligned}
& c_{0}=\alpha, \quad c_{1}=\frac{1}{2}, \quad c_{2}=1-\alpha, \\
& b_{0}=\frac{1}{6(1-2 \alpha)^{2}}, \quad b_{1}=1-2 b_{0}, \quad b_{2}=b_{0}, \\
& \bar{b}_{0}=b_{0}\left(1-c_{0}\right), \quad \bar{b}_{1}=b_{1}\left(1-c_{1}\right), \quad \bar{b}_{2}=b_{2}\left(1-c_{2}\right), \\
& \bar{a}_{1,0}=\frac{(1-4 \alpha)(1-2 \alpha)}{8(6 \alpha(\alpha-1)+1)}, \quad \bar{a}_{2,0}=2 \alpha(1-2 \alpha), \quad \bar{a}_{2,1}=\frac{(1-2 \alpha)(1-4 \alpha)}{2},
\end{aligned}
$$

$\alpha$ is a free parameter a maximal CFL of 3.939 is obtained for

$$
\alpha=\frac{1}{4\left(1+\cos \left(\frac{\pi}{9}\right)\right)} .
$$

Order $5(s=4)$ : A family of RKN schemes of order 5 with two parameters is given in [6]. A maximal CFL of 2.908 is obtained for

$$
\alpha=\frac{4}{11+\sqrt{16 \sqrt{10}-39}}, \beta=\frac{165 \alpha^{2}-195 \alpha+50-\sqrt{5\left(45 \alpha^{4}+90 \alpha^{3}-105 \alpha^{2}+36 \alpha-4\right)}}{225 \alpha^{2}-240 \alpha+60} .
$$

The $c_{i}$ are given as

$$
c_{0}=0, \quad c_{1}=\alpha, \quad c_{3}=\beta, \quad c_{2}=\frac{12-15(\alpha+\beta)+20 \alpha \beta}{15-20(\alpha+\beta)+30 \alpha \beta} .
$$

From order 6 to 10, the optimal coefficients for RKN schemes are new. They have been computed numerically, only, in the following subsections.

\subsubsection{Order $6(s=5)$}

A family with one parameter is given in [6]. Using Algorithm 1, we have obtained a maximal CFL of 3.089 for

$$
\alpha \approx 0.22918326
$$

The $c_{i}$ are given as

$$
c_{0}=0, \quad c_{1}=\alpha, \quad c_{2}=\frac{1}{2}, \quad c_{3}=1-\alpha, \quad c_{4}=1 .
$$

Another family with two parameters can also be constructed. The maximal CFL is also equal to 3.089 for this family. 


\subsubsection{Order $7(s=7)$}

A family of RKN schemes of order 7 with four free parameters is given in [6]. After optimization, we have obtained a maximal CFL of 7.0875 with the following parameters:

$$
\begin{array}{ll}
\alpha_{0}=0.110451398065702, & \alpha_{1}=0.173816271367107 \\
\alpha_{2}=0.459433163929695, & \alpha_{3}=0.652002232653235
\end{array}
$$

The coefficients $c_{i}$ are given by $c_{0}=0, c_{1}=\alpha_{0}, c_{2}=\alpha_{1}, c_{3}=\alpha_{2}, c_{4}=\alpha_{3}, c_{5}=\frac{-\frac{1}{7}+\frac{\sigma_{1}^{c}}{6}-\frac{\sigma_{2}^{c}}{5}+\frac{\sigma_{3}^{c}}{4}-\frac{\sigma_{4}^{c}}{3}}{-\frac{1}{6}+\frac{\sigma_{1}^{c}}{5}-\frac{\sigma_{2}^{c}}{4}+\frac{\sigma_{3}^{c}}{3}-\frac{\sigma_{4}^{c}}{2}}, c_{6}=1$.

\subsubsection{Order $8(s=8)$}

A family of RKN schemes of order 8 with four free parameters is given in [6]. A maximal CFL of 7.8525 is obtained with the following parameters

$$
\begin{gathered}
\alpha_{0}=0.135294127286225, \quad \alpha_{1}=0.24015308384744 \\
\alpha_{2}=0.453046953126355, \quad \alpha_{3}=0.695039606659698
\end{gathered}
$$

The coefficients $c_{i}$ are given by

$$
\begin{gathered}
c_{0}=0, \quad c_{1}=\frac{\alpha_{0}}{2}, \quad c_{2}=\alpha_{0}, \quad c_{3}=\alpha_{1}, \quad c_{4}=\alpha_{2}, \quad c_{5}=\alpha_{3} \\
c_{6}=\frac{-\frac{1}{8}+\frac{\sigma_{1}^{c}}{7}-\frac{\sigma_{2}^{c}}{6}+\frac{\sigma_{3}^{c}}{5}-\frac{\sigma_{4}^{c}}{4}+\frac{\sigma_{5}^{c}}{3}}{-\frac{1}{7}+\frac{\sigma_{1}^{c}}{6}-\frac{\sigma_{2}^{c}}{5}+\frac{\sigma_{3}^{c}}{4}-\frac{\sigma_{4}^{c}}{3}+\frac{\sigma_{5}^{c}}{2}}, \quad c_{7}=1 .
\end{gathered}
$$

\subsubsection{Order $10(s=11)$}

In [7], the author present a family of RKN schemes of order 10 with four free parameters $\left(b_{0}, b_{2}, b_{3}, r_{5}\right) . r_{5}$ is an additional free parameter that we have recognized during the construction of the family, it is defined as

$$
r_{5}=\sum_{i=0}^{s-1} b_{i} c_{i}^{3} \sum_{j=0}^{i-1} \bar{a}_{i j} c_{j}^{5}
$$

Following the work in [7] we denote the Gauss-Lobatto nodes $\gamma_{1}, \gamma_{2}, \gamma_{3}, \gamma_{4}$ : 


$$
\begin{cases}\gamma_{1}=\frac{1}{2}\left(1-\sqrt{\frac{7+2 \sqrt{7}}{21}}\right), & \gamma_{4}=1-\gamma_{1}, \\ \gamma_{2}=\frac{1}{2}\left(1-\sqrt{\frac{7-2 \sqrt{7}}{21}}\right), & \gamma_{3}=1-\gamma_{2} .\end{cases}
$$

Among the 24 permutations choice possible for $\left(c_{3}, c_{4}, c_{5}, c_{6}\right)$, the CFL is maximal for the following permutation

$$
\left(c_{3}, c_{4}, c_{5}, c_{6}\right)=\left(\gamma_{4}, \gamma_{3}, \gamma_{1}, \gamma_{2}\right) .
$$

The other $c_{i}$ are given by

$c_{0}=0, \quad c_{2}=\frac{c_{4}\left(3 c_{4}-5 c_{3}\right)}{5 c_{4}-10 c_{3}}, \quad c_{1}=\frac{c_{2}}{2}, \quad c_{7}=c_{3}, \quad c_{8}=c_{2}, \quad c_{9}=0, \quad c_{10}=1$.

For this permutation, we have obtained a maximal CFL of 4.7527 with the following parameter

$$
r_{5}=0.0021632268153138
$$

The CFL is maximal for this permutation only, it is strictly lower for other permutations. For other parameters, we can choose the values proposed by Hairer in [7]:

$$
b_{0}=0, \quad b_{2}=-0.1, \quad b_{3}=0,
$$

since the CFL does not depend on these three parameters.

\subsection{Efficiency and numerical results}

\subsubsection{Efficiency}

Let $s$ be the number of stages of the RKN scheme. The efficiency is given as:

$$
\text { Efficiency }=\frac{\text { CFL number }}{2 s} .
$$

An optimal scheme is a scheme such that the efficiency is maximal. Since $s$ is constant, the efficiency is maximal for a maximal CFL number. In Table 1.1, we have written the efficiency of the different RKN schemes. We observe that the orders 7

Table 1.1 Efficiency of optimized Runge-Kutta-Nyström schemes of different orders

\begin{tabular}{|c|c|c|c|c|c|c|c|c|}
\hline Order & 2 & 3 & 4 & 5 & 6 & 7 & 8 & 10 \\
\hline Efficiency & $100 \%$ & $62.5 \%$ & $65.7 \%$ & $36.4 \%$ & $30.9 \%$ & $50.6 \%$ & $49.1 \%$ & $21.6 \%$ \\
\hline
\end{tabular}


and 8 are attractive since they have a correct efficiency (about $50 \%$ ). It is important to have optimal schemes when the ODE to be solved is stiff, i.e. when the obtained accuracy is satisfactory when the maximal time step is chosen. However, for low orders (such as 2,3 ), the accuracy is usually poor such that the time step must be chosen much smaller than the maximal time step to obtain a good solution.

\subsubsection{Numerical results}

Using the higher order-finite element code Montjoie, we applied the RKN schemes to solve the non-linear Maxwell's equations in 1-D, namely:

$$
\left\{\begin{array}{l}
\frac{\varepsilon_{\infty}}{c^{2}} \frac{\partial^{2} E}{\partial t^{2}}+\frac{1}{c^{2}} \frac{\partial^{2}}{\partial t^{2}}\left(\sum_{k} P_{k}\right)-\frac{\partial^{2} E}{\partial z^{2}}+\frac{\rho}{c^{2}} \frac{\partial^{2}}{\partial t^{2}}\left(|E|^{2} E\right)=0 \\
\frac{1}{\omega_{k}^{2}} \frac{\partial^{2} P_{k}}{\partial t^{2}}+P_{k}=v_{k} E \\
E(z, t=0)=\frac{\partial E}{\partial t}(z, t=0)=0 \\
E(z=0, t)=\text { Given impulsion }
\end{array}\right.
$$

Here the electric field is searched as a complex field:

$$
E=E_{x}+i E_{y}
$$

where $E_{x}$ and $E_{y}$ are x and y-components of the electric field. $P_{k}$ is the polarization, $\varepsilon_{\infty}, c, \rho, v_{k}, \omega_{k}$ are physical constants. We take the constants corresponding to silica:

$$
\varepsilon_{\infty}=1, c=299792458, v_{0}=0.6961663, v_{1}=0.4079426, v_{2}=0.8974794
$$

$\omega_{0}=\frac{2 \pi c}{0.0684043 \cdot 10^{-6}}, \omega_{1}=\frac{2 \pi c}{0.1162414 \cdot 10^{-6}}, \omega_{2}=\frac{2 \pi c}{9.896161 \cdot 10^{-6}}, \gamma=10^{-33}$.

The impulsion is centered at $\lambda_{0}=1.053 \mu \mathrm{m}$ with a Gaussian envelope and a circular polarization:

$$
\text { Given impulsion }=E_{0} e^{-\frac{1}{2}\left(\frac{t-T_{\max }}{\tau}\right)^{2}} e^{i \omega_{L} t}
$$

where

$$
\omega_{L}=\frac{2 \pi c}{1.053 \cdot 10^{-6}}, T_{\max }=6 \cdot 10^{-14}, \tau=\frac{2}{2 \sqrt{2 \log 2}} \cdot 10^{-14}, E_{0}=10^{9} .
$$


The computational domain is the 1 -D interval $\Omega=\left[0,1.5 \cdot 10^{-4}\right]$, a Neumann boundary condition is set on the right extremity. 1-D finite elements are used to discretize these equations:

$$
E \in V_{h}=\left\{u \in H^{1}(\Omega) \text { such that }\left.u\right|_{\left[z_{i}, z_{i+1}\right]} \in \mathbb{Q}_{10}\right\}
$$

where $\left(z_{i}\right)_{0 \leq i \leq 250}$ are a regular subdivision of the computational domain $\Omega$. The mesh contains 250 elements (i.e. 2501 degrees of freedom), the numerical error due to the space discretization is around $10^{-6}$ (the domain contains more than 200 wavelengths). After space discretization, the system can be written in the form

$$
y^{\prime \prime}=f(t, y)
$$

by using the displacement as unknown

$$
D=\varepsilon_{\infty} E+\left(\sum_{k} P_{k}\right)+\rho|E|^{2} E .
$$

The electric field $E$ is recovered from $D$ by solving the non-linear equation written above for each degree of freedom. This equation is solved with a Newton's method, two or three iterations are sufficient to get machine precision accuracy. Gauss-Lobatto points are used both for interpolation (for the discretization of $V_{h}$ ) and quadrature, leading to a diagonal mass matrix. As a result the computation of $f(t, y)$ is explicit, it does not involve any solution of a linear system. The electric field is propagated from $t=0$ until $t=5 \cdot 10^{-11}$, in Figure 1.3, the solution is plotted at two different times. The solution at the final time $t=5 \cdot 10^{-11}$ is compared with a reference solution computed with a small time step (with tenth order RKN scheme). We try to reach an error of $0.01 \%$ for each scheme in order to compare the efficiency. In Table 1.2, the computational time needed to obtain this accuracy
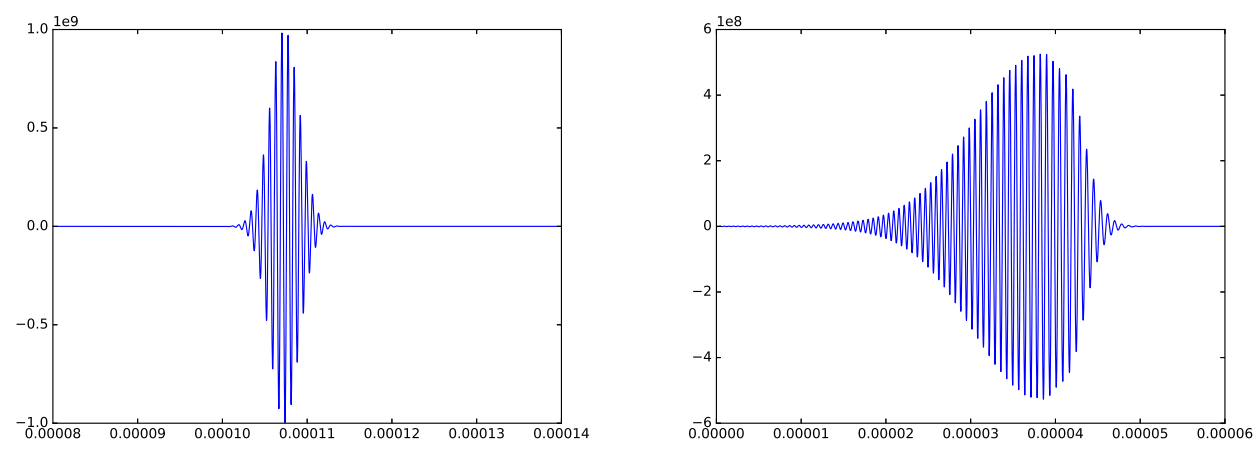

Fig. 1.3 Electric field $E_{x}$ for $t=10^{-12}$ and $t=5 \cdot 10^{-11}$ 
Table 1.2 Computational time needed to reach an accuracy of $0.01 \%$ for different orders of RKN schemes.

\begin{tabular}{|c|c|c|c|c|c|c|c|c|}
\hline Order & 2 & 3 & 4 & 5 & 6 & 7 & 8 & 10 \\
\hline Time & $14164 \mathrm{~s}$ & $730 \mathrm{~s}$ & $144 \mathrm{~s}$ & $60.9 \mathrm{~s}$ & $70.8 \mathrm{~s}$ & $43.2 \mathrm{~s}$ & $44.8 \mathrm{~s}$ & $103 \mathrm{~s}$ \\
Error & $1.0 \cdot 10^{-4}$ & $1.0 \cdot 10^{-4}$ & $1.0 \cdot 10^{-4}$ & $1.5 \cdot 10^{-6}$ & $7.3 \cdot 10^{-7}$ & $6.5 \cdot 10^{-7}$ & $1.1 \cdot 10^{-7}$ & $2.0 \cdot 10^{-10}$ \\
$\Delta t$ & $9.1 \cdot 10^{-20}$ & $2.9 \cdot 10^{-18}$ & $2.0 \cdot 10^{-17}$ & $6.2 \cdot 10^{-17}$ & $6.6 \cdot 10^{-17}$ & $1.5 \cdot 10^{-16}$ & $1.7 \cdot 10^{-16}$ & $1.0 \cdot 10^{-16}$ \\
\hline
\end{tabular}

is given for each optimized RKN scheme. The simulations are performed in parallel on 20 cores on an Intel-Xeon (2 Dodeca-core Haswell E5-2680, 2.5 Ghz). From order 5, we are using the maximal time step allowed (because of the restrictive CFL), that's why the error is below $0.01 \%$ for these orders. For orders 2,3 and 4 , the time step required to obtain an error of $0.01 \%$ is much smaller than the maximal time step, that's why they are less efficient. In this case, we observe that low order schemes $(2,3,4)$ are limited by the accuracy whereas high-order schemes are limited by the CFL. We see that RKN schemes of order 7 or 8 are the most efficient for this problem while order 10 is not very efficient because of its small CFL.

\subsection{Conclusion}

In this work we have proposed an algorithm to compute the CFL number of a RKN scheme. By using this algorithm, we have computed optimal coefficients for RKN schemes of order $6,7,8$ and 10 that maximize the CFL number. The numerical results we presented show the practical interest of these schemes, in paticular order 7 and 8. In fact, we have observed that lower order schemes are limited by the accuracy while the scheme of order 10 is less efficient due to its small CFL. We think that the efficiency can be further increased by adding more stages ([2], [3]).

\section{References}

1. E. Hairer, Syvert P. Norsett and G. Wanner, "Solving ordinary differential equations I - Nonstiff problems", Springer, (2008)

2. J. Neigemann, R. Diehl and K. Bush, "Efficient low-storage Runge-Kutta schemes with optimized stability regions", Journal of Computational Physics. 231 , pp 364-372 (2012)

3. P. Joly and J-C Gilbert, "Higher order time stepping for second order hyperbolic problems and optimal CFL conditions", Computational Methods in Applied Sciences. 16, 67-93 (2008)

4. M. Chawla and S. Sharma, "Families of fifth-order Nyström methods for $y^{\prime \prime}=f(x, y)$ and intervals of periodicity", Computing. 26, pp 247-256 (1981)

5. M. Chawla and S. Sharma, "Interval of periodicity and absolute stability of explicit Nyström methods for $y^{\prime \prime}=f(x, y)$ ", BIT. 21, 455-464 (1981)

6. E. Hairer, "Méthodes de Nyström pour l'équation différentielle $y^{\prime \prime}=f(x, y)$ ", Numer. Math.27, 283-300 (1977)

7. E. Hairer, "A one-step method of order 10 for $y^{\prime \prime}(t)=f(t, y)$ “, IMA J. Numer. Anal. 2, 83-94 (1982) 